\title{
Assessment of basal insulin adherence using 2 methodologies among Texas Medicaid enrollees with type 2 diabetes
}

Hanxi Zhang; Jamie C Barner, PhD; Leticia R Moczygemba, PhD; and Karen L Rascati, PhD

\section{What is already known about this subject}

- Although insulin adherence is critical in diabetes management, suboptimal adherence to insulin therapy has been reported in various settings.

- Second-generation long-acting (SGLA) insulin may have improved adherence because of its improved safety and regimen convenience, yet little realworld evidence is available.

- Days supply of insulin recorded in administrative claims are often inaccurate, making it challenging to derive insulin adherence from the claims data; adjusted medication possession ratio (aMPR) has been used to account for inaccuracies in recorded days supply.

\section{ABSTRACT \\ BACKGROUND: Basal insulin is often recom- mended as the initial therapy for patients with type 2 diabetes who require insulin treatment. Adequate adherence is critical to diabetes management, yet suboptimal insulin adherence has been reported. Second-generation long-acting (SGLA) insulin has higher dosing flexibility and lower hypo- glycemia risk and may improve adherence. However, little is known regarding adher- ence to SGLA insulin and how adherence to SGLA insulin compares with intermediate- acting neutral protamine Hagedorn (NPH) and first-generation long-acting (FGLA)}

\begin{abstract}
What this study adds
- Whether using MPR or aMPR, there was no significant difference in adherence between SGLA insulin and other basal insulins after controlling for covariates among Texas Medicaid enrollees with type 2 diabetes.

- With aMPR, the same adjustment is applied to all patients using the same insulin product, but without individuallevel adjustments, associations between adherence, measured using either MPR or aMPR, and factors other than insulin type may be confounded by dispensing patterns and individual insulin needs.

- Reporting MPR and aMPR results is recommended, since aMPR may overestimate adherence in general and bias adherence comparisons despite having the advantage over MPR in accounting for varying package sizes of insulin products.
\end{abstract}

insulin. Measurement of insulin adherence is challenging because of the inaccuracies of recorded days supply of insulin, and traditional medication possession ratio (MPR) may be negatively affected. Adjusted MPR (aMPR) has been developed in an effort to address this issue.

OBJECTIVE: To examine the unadjusted and adjusted associations between basal insulin type and adherence to basal insulin using MPR and aMPR.

METHODS: This retrospective database study used Texas Medicaid prescription claims from January 1, 2014, through June 30, 2017. The index date was the date of the first basal

\begin{abstract}
Author affiliations
Hanxi Zhang; Jamie C Barner, PhD; Leticia R Moczygemba, PhD; and Karen L Rascati, $\mathrm{PhD}$, Health Outcomes Division, College of Pharmacy, University of Texas at Austin.
\end{abstract}

\section{AUTHOR CORRESPONDENCE: \\ Jamie C Barner, 512.471.5612, \\ jbarner@austin.utexas.edu}

insulin prescription without the same prescription 6 months before (pre-index), and all patients were followed for 12 months (post-index). Patients aged 18-63 years with $\geq 1$ pre-index prescription of an oral hypoglycemia agent (OHA) or a glucagon-like peptide-1 receptor agonist (GLP-1 RA), without any post-index prescription of premixed insulin or a basal insulin different from index insulin, and with continuous enrollment throughout the pre- and post-index periods, were included. The dependent variable was basal insulin adherence over 12 months, measured using MPR and aMPR. Unadjusted and adjusted adherence comparisons were conducted by basal (background) insulin 
type (NPH, FGLA, and SGLA). Covariates included age, gender, baseline use of basal insulins and comorbid medications, total number of medications, OHA adherence, post-index number of OHAs, and use of bolus insulins and GLP-1 RAs. Analysis of variance, chi-square tests, and multiple logistic regression analyses were performed.

RESULTS: Of the 5,034 patients included, NPH, FGLA, and SGLA insulin users accounted for $3.7 \%, 89.8 \%$, and $6.5 \%$, respectively. The overall mean (SD) age was 50.9 (9.9) years, and $65.9 \%$ were female. In the unadjusted bivariate analyses, SGLA insulin users had significantly higher adherence, using either MPR (SGLA 0.68 [0.25] vs. FGLA 0.59 [0.27] vs. NPH 0.55 [0.27]; $P<0.0001)$ or aMPR (0.83 [0.23] vs. 0.78 [0.26] vs. 0.73 [0.28]; $P=0.0001)$. After controlling for covariates, insulin type was not significantly associated with the likelihood of being adherent (MPR or $\mathrm{aMPR} \geq 0.8$ ) using either measure.

CONCLUSIONS: Adherence to SGLA insulin was not different from adherence to other basal insulins after controlling for patient characteristics. Yet, MPR and aMPR have limitations and warrant further confirmation of the study findings. Before new adherence measures for insulin therapy are developed, MPR and aMPR should be used with caution.

Despite a leveling off in the prevalence of diabetes since around $2008{ }^{1-3}$ diabetes remains prevalent in the United States. Based on the Centers for Disease Control and Prevention, the crude prevalence of all diabetes in the United States was $10.5 \%$ in 2018 , with the vast majority (90\%-95\%) being type 2 diabetes (T2D). ${ }^{4}$ Diabetes imposes a substantial economic burden on society, costing more than $\$ 300$ billion in the United States in 2017 alone. $^{5}$

Although oral hypoglycemia agents (OHAs), such as metformin, are typically first-line therapy for patients with $\mathrm{T} 2 \mathrm{D}$, as the disease progresses, some T2D patients require insulin therapy for glycemic control. ${ }^{6}$ In the United States, an estimated $29.1 \%$ of patients with diabetes of any type were treated with insulin, ${ }^{7}$ and $10.9 \%$ of adult patients were initiated on insulin therapy within 1 year of their diagnosis of diabetes. ${ }^{4}$ According to guidelines, basal insulin alone may be used as the initial insulin therapy. ${ }^{6,8}$

Basal insulin therapy mimics the basal (or background) component of the body's normal insulin secretion. ${ }^{9}$ The optimal action profile of a basal insulin, therefore, should be flat without any pronounced peak and have a long duration. Currently available basal insulins fall into 3 types: intermediate-acting neutral protamine Hagedorn (NPH) insulin, first-generation long-acting (FGLA) insulin analogues (i.e., glargine U100 and detemir), and second-generation long-acting (SGLA) insulin analogues (i.e., glargine U300, degludec U100, and degludec U200). All forms of NPH and
FGLA insulins are available in pens and vials, whereas SGLA insulins, all forms of which were approved in 2015, are currently available in pens only (except for degludec U100, which is available in pens and vials). While NPH insulin requires a twice-daily dosing regimen because of its short duration of action and has a peak effect, FGLA insulins may allow for once-daily dosing for some patients and have less pronounced peak effects. ${ }^{10}$ Compared with FGLA insulins, SGLA insulins have even longer action profiles and more constant insulin levels, which may translate into greater dosing flexibility and lower hypoglycemia risk. ${ }^{10}$ With improved regimen convenience and safety, SGLA insulin holds the potential of improving patient adherence to basal insulin therapy.

Adherence is important for patients on insulin therapy to achieve and maintain treatment goals, yet nonadherence rates ranging from $20 \%$ to $38 \%$ have been reported in previous studies. ${ }^{11}$ In particular, Medicaid enrollees may be at an even higher risk of nonadherence. ${ }^{12}$ Lack of insulin regimen flexibility, burden of multiple injections, and risk of hypoglycemia are some identified barriers to insulin adherence. $^{13}$ Regarding the newest type of basal insulin, SGLA insulin, little is known about patient adherence and how it compares with NPH and FGLA insulin adherence.

Challenges, however, exist with insulin adherence measurement. Although medication possession ratio (MPR) and proportion of days covered (PDC) are the most commonly used measures of insulin adherence, both measures may be inaccurate. ${ }^{14}$ The calculations of both measures rely on recorded days supply of insulin, and it has been widely recognized that, for insulin therapy, there is often a lack of congruency between the recorded and "true" days supply. ${ }^{14}$ To address this issue, a method called adjusted MPR (aMPR) was developed..$^{15}$ This data-driven method aims to mitigate the effect of inaccurately recorded days supply by multiplying the traditional MPR by a ratio of mean (or median) days between refills divided by mean (or median) recorded days supply among all patients on the same insulin. ${ }^{15,16}$ Most commonly, aMPR has been used in previous studies when there is a need to compare adherence across different insulin products, ${ }^{15-20}$ particularly when the products have different package sizes and/or delivery devices. When both MPR and aMPR results were reported, aMPR was almost always greater than MPR (i.e., mean or median between-refill time was almost always longer than mean or median days supply as recorded) with very few exceptions. Previous studies using MPR and aMPR have shown mixed results regarding the consistency of adherence comparison results. 15,18,19,21,22

Most of the existing studies that have assessed basal insulin adherence were conducted in commercially insured or Medicare populations using NPH and/or FGLA insulin, ${ }^{15-23}$ 
so little is known about insulin adherence among patients who are Medicaid beneficiaries or adherence to SGLA insulin. This study aimed to assess unadjusted and adjusted associations between basal insulin adherence and insulin type (i.e., NPH, FGLA, and SGLA insulin) among Texas Medicaid beneficiaries. Because of the lack of a gold standard in insulin adherence measurement, both MPR and aMPR were used in the adherence measurement.

\section{Methods}

\section{DATA SOURCE AND STUDY POPULATION}

This was a retrospective study using Texas Medicaid prescription claims data from January 1, 2014, to June 30, 2017. The index date was the date of the first prescription of basal insulin (i.e., NPH, glargine U100, detemir, glargine U300, or degludec U200) without a previous prescription for the same insulin 6 months before (Figure 1). ${ }^{24}$ Patients with T2D who were naive to their index basal insulin were included (i.e., eligible patients included insulin naive and experienced patients, which we believe would better reflect the realworld population initiated on any of the studied basal insulin, compared with a sample of insulin-naive patients).

Specifically, the inclusion criteria were as follows: (a) aged 18-63 years (to avoid Medicare-Medicaid dual eligibles); (b) had at least 2 prescriptions (index prescription included) of the same basal insulin during 12 months after the index date (i.e., post-index period) ${ }^{25}$; (c) had at least 1 prescription of any OHA or glucagon-like peptide-1 receptor agonist (GLP-1 RA) 6 months before the index date (i.e., pre-index period; to ensure T2D; guidelines recommend OHAs as initial therapy for T2D patients and then consideration of injectable GLP-1 RAs before insulin initiation ${ }^{6}$ ); and (d) continuously enrolled during the 6-month pre-index and 12-month post-index periods.

Patients prescribed degludec U100 at index were not included, since degludec U100 was the only nonconcentrated form of SGLA insulin, so its users may not represent typical SGLA insulin users. Additionally, patients who were prescribed premixed insulin or a basal insulin different from index insulin during the post-index period were also excluded, since adherence to the index insulin may not accurately capture these patients' adherence to their prescribed insulin therapies.

\section{STUDY VARIABLES}

The outcome, adherence to index basal insulin, was measured using MPR and aMPR over the 12-month post-index period. MPR was calculated by first summing up the recorded days supply of all claims for index insulin during the postindex period and then dividing the sum by 365 days. ${ }^{25}$

$$
M P R=\frac{\begin{array}{l}
\text { Sum of the days supply of index } \\
\text { insulin in the post-index period }
\end{array}}{\text { Number of days in the post-index period }}
$$

To calculate aMPR, the MPR of each patient was multiplied by an adjustment factor. ${ }^{15}$ The adjustment factor was calculated for each basal insulin product type as the ratio of the average number of days between refills of 1 basal insulin product divided by the average recorded days supply for the same insulin product.

$$
\begin{gathered}
\text { Average days between refills for } \\
\text { index insulin in the post-index period } \\
\text { amPR }=\mathrm{MPR} \times \frac{\text { among all patients on the same insulin }}{\text { Average days supply of index insulin }} \\
\begin{array}{c}
\text { in the post-index period among all } \\
\text { patients on the same insulin }
\end{array}
\end{gathered}
$$

Compared with the average recorded days supply, which is often close to 30 days and lacks variance ${ }^{15}$ the average days between refills may better reflect the real-world balance achieved between average insulin supply and the average patient's insulin needs within patient groups. Thus, applying the adjustment factor may enable aMPR to be superior to traditional MPR, when the average days between refills is a better estimate of "true" average days supplied than the average recorded days supply.

Both MPR and aMPR were truncated at 1 , and a cutoff of $\geq 0.8$ was used for both measures in identifying adherence. Sensitivity analyses were performed, where each adjustment factor was calculated as the median number of days between refills of a basal insulin product divided by the median recorded days supply for the same product.

The main independent variable was index basal insulin type, with 3 categories: NPH, FGLA (i.e., glargine U100 and detemir), and concentrated SGLA insulin (i.e., glargine U300 and degludec U200). Covariates included demographic characteristics at index (i.e., age and gender); clinical characteristics during the pre-index period and at baseline (i.e., pre-index basal insulin use, OHA adherence, medication use for comorbid hypertension and dyslipidemia, and baseline total number of medications); and clinical characteristics during the post-index period (i.e., number of OHAs, bolus insulin use, and GLP-1 RA use). Delivery type (vial vs. pen) was not included as a covariate, since both SGLA insulin products included in this study were delivered in pen formulation only.

\section{STATISTICAL ANALYSES}

Descriptive statistics were calculated for demographic and clinical characteristics. Unadjusted bivariate comparisons 


\section{FIGURE 1 Study Design}

Cohort Entry Date
(First Rx of NPH, glargine U100, detemir, glargine U300, or degludec

U200 insulin between January 1, 2014, and June 30, 2017)

Day 0

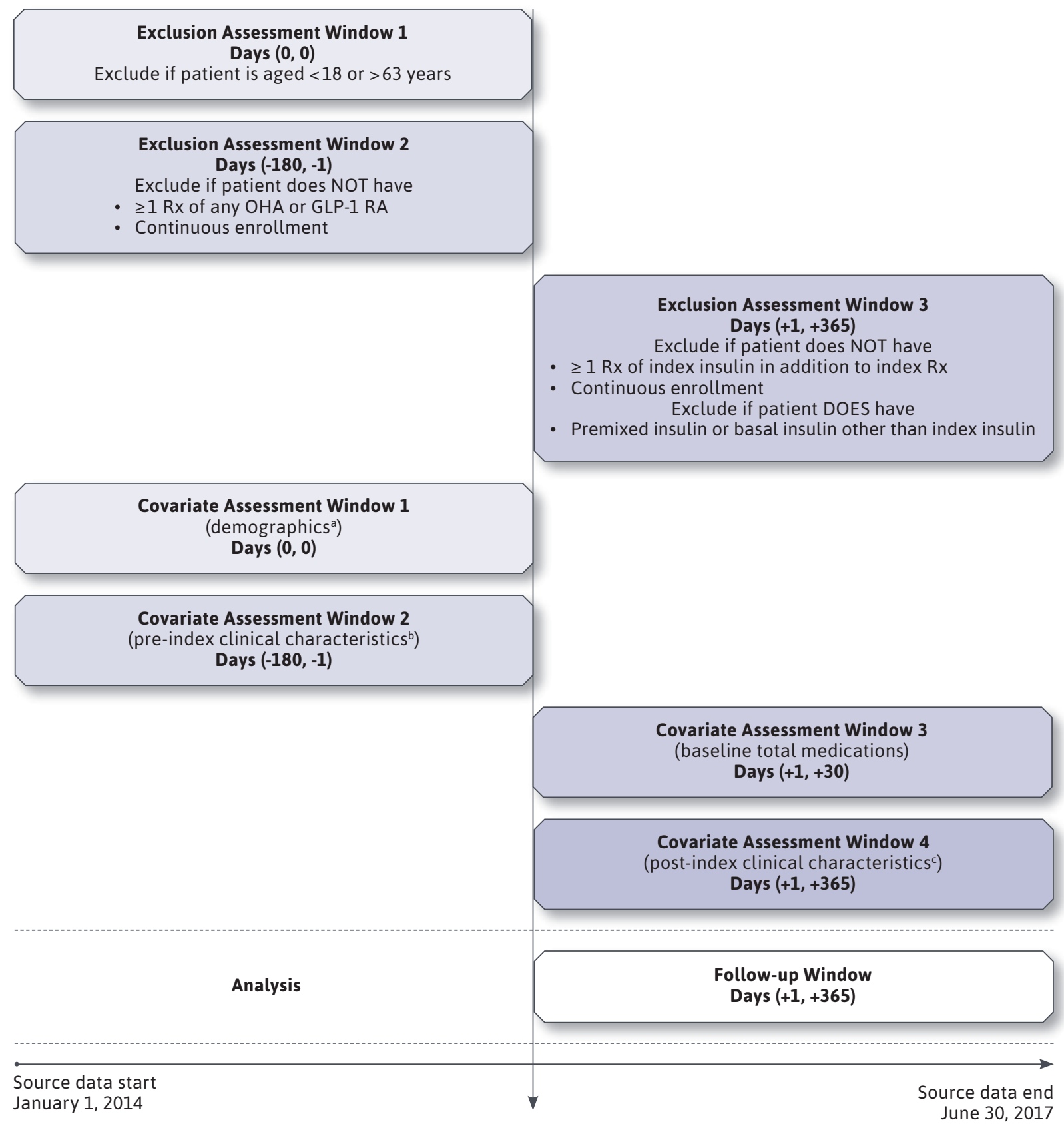

${ }^{a}$ Demographics included age and gender.

${ }^{b}$ Pre-index clinical characteristics included use of other basal insulin, OHA adherence, and use of comorbid medications.

cPost-index clinical characteristics included number of OHAs, use of bolus insulin, and use of GLP-1 RA.

GLP-1 RA=glucagon-like peptide-1 receptor agonist; $N P H=$ neutral protamine Hagedorn; $O H A=$ oral hypoglycemic agent; $R x=$ prescription . 


\section{FIGURE 2 Patient Attrition}

Patients with any basal insulin prescription during January 1, 2014-June 30, 2017 $\mathrm{N}=57,974$

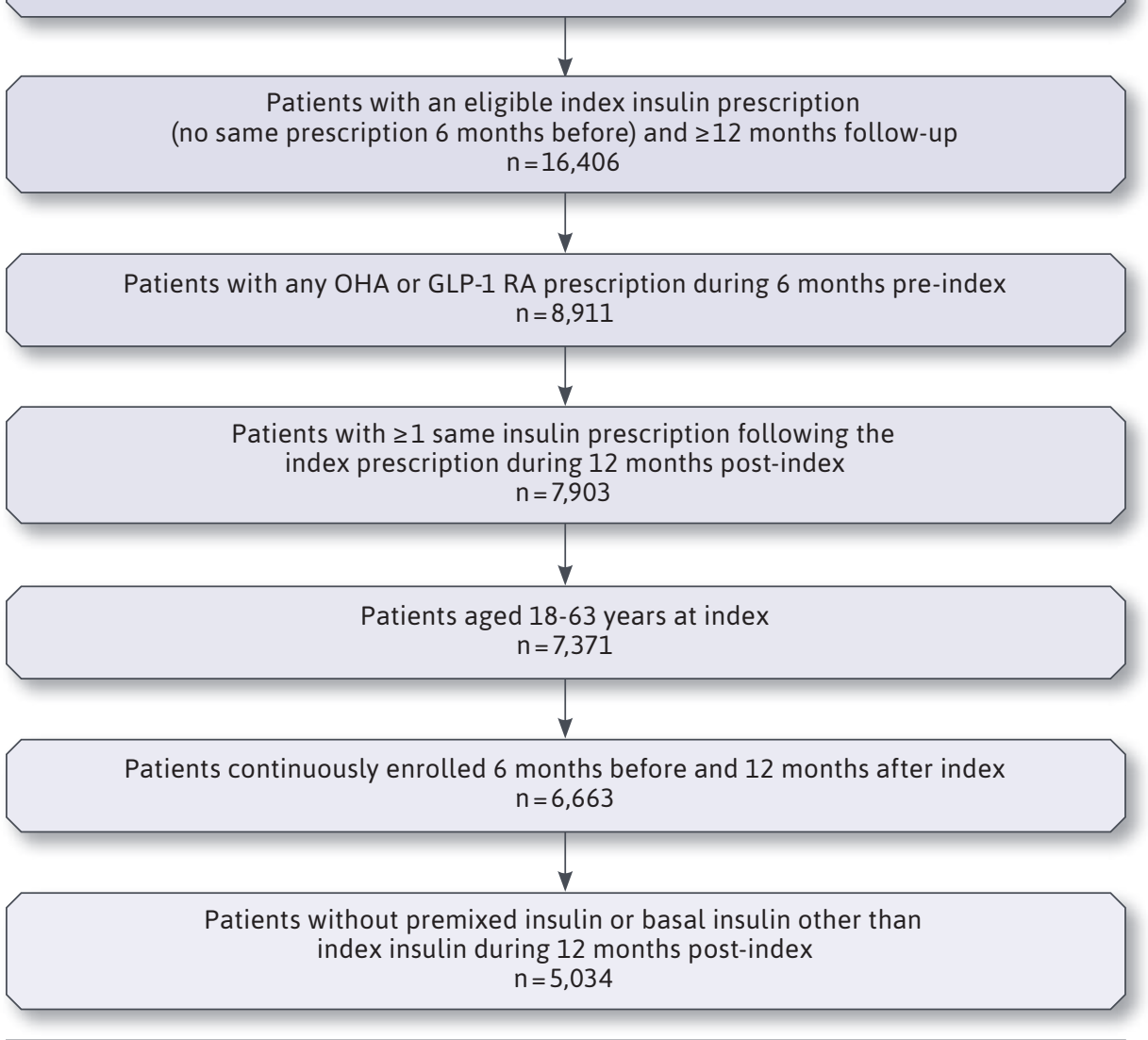

GLP-1 RA = glucagon-like peptide-1 receptor agonist; $\mathrm{OHA}=$ oral hypoglycemic agent.

of adherence by basal insulin type were performed using analysis of variance. Multiple logistic regression analyses were conducted to model the odds of being adherent (i.e., MPR $\geq 0.8$ or $\mathrm{aMPR} \geq 0.8$ ) when controlling for demographic and clinical covariates. Because notable imbalances were observed in certain covariates (e.g., previous use of basal insulin) by index insulin type, the traditional covariate adjustment may be subject to model extrapolation. Therefore, sensitivity analyses combining regression adjustment and weighting based on propensity scores were performed (see methods and results in Supplementary Tables 1 and 2, available in online article). Additional sensitivity analyses were performed to assess whether results would differ in insulin-naive and experienced patients by rerunning the unweighted logistic models (with pre-index use of basal insulin removed from the covariates) separately for the 2 types of patients.

All statistical tests were two-sided with an a priori significance level set at $\mathrm{P}<0.05$. SAS version 9.4 (SAS Institute, Cary, NC) was used for all statistical analyses. As determined by the Institutional Review Board (IRB) of the University of Texas at Austin, this study was exempt from IRB review.

\section{Results}

After applying the inclusion and exclusion criteria, 5,034 patients were included (Figure 2). Of these, 187 (3.7\%) received NPH; 4,522 (89.8\%) received FGLA; and 325 (6.5\%) received SGLA insulin at index. Overall, the mean age (SD) was 50.9 (9.9) years, and the majority was female (65.9\%). Demographic and clinical characteristics at baseline were compared by index insulin type (Table 1). NPH insulin users were significantly $(\mathrm{P}<0.0001)$ younger (46.2 [13.1] years) on average compared with NPH (51.1 [9.7] years) and FGLA insulin users (51.0 [10.0] years). The total number of medications at baseline were higher for SGLA insulin users versus NPH and FGLA insulin users $(P=0.0003)$.

During the pre-index period, SGLA insulin users were also more likely to have previous experience with insulin and injectable GLP-1 RAs, in addition to having better adherence to OHAs (all $P$ values $<0.0001$ ). During the postindex period, SGLA insulin users had more distinct OHAs and were more likely to have injectable GLP-1 RAs compared with NPH and FGLA insulin users (all $P$ values $<0.0001$ ). The proportion of post-index bolus insulin use also varied by index insulin type $(P=0.0014)$, with SGLA insulin users having a proportion higher than FGLA insulin users but similar to NPH insulin users.

During the 12-month follow-up, the bivariate analyses (Table 2) showed that SGLA insulin users had the highest mean (SD) MPR (NPH: 0.55 [0.27], FGLA: 0.59 [0.27], SGLA: 0.68 [0.25]; $\mathrm{P}<0.0001$ ); mean (SD) aMPR (NPH: 0.73 [0.28], FGLA: 0.78 [0.26], SGLA: 0.83 [0.23]; $\mathrm{P}=0.0001$ ); proportion of patients having $\mathrm{MPR} \geq 80 \%$ (NPH: 21.4\%, FGLA: 27.6\%, SGLA: 39.7\%; 


\section{TABLE 1 Comparison of Baseline Demographic and Clinical Characteristics Among NPH, First-, and Second-Generation Long-Acting Insulin Users $(N=5,034)$}

\begin{tabular}{|c|c|c|c|c|}
\hline Characteristics & $\begin{array}{c}\text { Intermediate- } \\
\text { Acting } \\
\text { (NPH) } \\
\text { (n=187) }\end{array}$ & $\begin{array}{c}\text { First- } \\
\text { Generation } \\
\text { Long-Acting } \\
(\mathrm{n}=\mathbf{4 , 5 2 2 )}\end{array}$ & $\begin{array}{c}\text { Second- } \\
\text { Generation } \\
\text { Long-Acting } \\
(n=325)\end{array}$ & P Value ${ }^{a}$ \\
\hline \multicolumn{5}{|l|}{ Baseline characteristics } \\
\hline Age, mean (SD) & $46.2(13.1)^{b}$ & $(9.7)^{\mathrm{c}}$ & $51.0(10.0)^{c}$ & $<0.0001$ \\
\hline \multicolumn{5}{|l|}{ Age, years, n (\%) } \\
\hline $18-39$ & $67(35.8)^{\mathrm{b}}$ & $617(13.6)^{c}$ & $44(13.5)^{c}$ & \multirow{4}{*}{$<0.0001$} \\
\hline $40-49$ & $22(11.8)$ & $958 \quad(21.2)$ & $69(21.2)$ & \\
\hline $50-59$ & $70 \quad(37.4)$ & $2,051 \quad(45.4)$ & $147 \quad(45.2)$ & \\
\hline $60-63$ & $28(15.0)$ & $896 \quad(19.8)$ & $65(20.0)$ & \\
\hline Females, n (\%) & $123(66.8)$ & $2,981 \quad(66.0)$ & $215(66.2)$ & 0.9953 \\
\hline Vial delivery, n (\%)e & 174 (93.1) & $1,299 \quad(28.7)$ & $(0.0)$ & $<0.0001$ \\
\hline Total number of medications, mean (SD) & $9.3 \quad(4.9)^{\mathrm{b}}$ & $10.0 \quad(5.1)^{c}$ & $(5.6)^{d}$ & 0.0003 \\
\hline Number of OHAs, mean (SD) & $(0.8)$ & $(0.8)$ & $(0.9)$ & 0.0641 \\
\hline Use of other insulins (yes/no), n (\%) & $66(35.3)^{b}$ & $1,874(41.4)^{b}$ & $258(79.4)^{c}$ & $<0.0001$ \\
\hline Use of basal insulins (yes/no), n (\%) & $34(18.2)^{\mathrm{b}}$ & $1,016(22.5)^{b}$ & $215(66.2)^{c}$ & $<0.0001$ \\
\hline Use of GLP-1 RA (yes/no), n (\%) & $5 \quad(2.7)^{\mathrm{b}}$ & $(7.1)^{b}$ & $57(17.5)^{c}$ & $<0.0001$ \\
\hline Use of medications for comorbid hypertension and dyslipidemia (yes/no), n (\%) & $91(48.7)$ & $2,366 \quad(52.3)$ & $187(57.5)$ & 0.1070 \\
\hline OHA adherence, mean (SD) ${ }^{\dagger}$ & $0.47(0.26)^{\mathrm{b}}$ & $0.56(0.26)^{c}$ & $0.62(0.25)^{d}$ & $<0.0001$ \\
\hline \multicolumn{5}{|l|}{ Post-index characteristics } \\
\hline Number of OHAs, mean (SD) & $1.3 \quad(1.0)^{\mathrm{b}}$ & $(1.0)^{c}$ & $(1.1)^{d}$ & $<0.0001$ \\
\hline Use of bolus insulin (yes/no), n (\%) & $99(52.9)^{b, c}$ & $2,191(48.5)^{b}$ & $190(58.5)^{c}$ & 0.0014 \\
\hline Use of GLP-1 RA (yes/no), n (\%) & $(3.7)^{\mathrm{b}}$ & $504(11.2)^{c}$ & $88(27.1)^{d}$ & $<0.0001$ \\
\hline \multicolumn{5}{|c|}{$\begin{array}{l}\text { aP values were based on analysis of variance for continuous and count variables and on chi-square tests for categorical variables. } \\
b, c, d \text { Means (or proportions) with the same letter were not significantly different (Duncan's multiple range test or chi-square test with type } 1 \text { error correct } \\
\text { Bonferroni). } \\
\text { 'Refers to delivery type of index insulin (vial vs. pen); chi-square test was performed between NPH and first-generation long-acting insulin users only. } \\
\text { fOHA adherence was measured using proportion of days covered. Patients were allowed to be on more than } 1 \mathrm{OHA} \text {. } \\
\text { GLP-1 RA=glucagon-like peptide-1 receptor agonist; } N P H=\text { neutral protamine Hagedorn; } O H A=\text { oral hypoglycemic agent; SD=standard deviation. }\end{array}$} \\
\hline
\end{tabular}

$\mathrm{P}<0.0001)$; and proportion of patients having aMPR $\geq 80 \%$ (NPH: 49.2\%, FGLA: 60.0\%, SGLA: 67.1\%; P=0.0004).

Table 3 shows the adjusted odds ratios (ORs) of adherence based on MPR and aMPR, respectively, with both using a cutoff of $80 \%$. Index insulin type was not significantly associated with the odds of either MPR or aMPR. Regarding covariates, in both MPR and aMPR analyses, patients who had used basal insulin, who had better adherence to OHAs, and who had a higher total number of medications at baseline were more likely to be adherent to index basal insulin. Patients who had more OHAs, who used any bolus insulin, and who used any GLP-1RA during the post-index period were also more likely to be adherent.

\section{SENSITIVITY ANALYSES}

Similar results were obtained in the sensitivity analyses where the adjustment factors for aMPR were calculated using medians instead of means. The main findings were generally consistent with the results of the sensitivity analyses using propensity score weighting (PSW; Supplementary Table 2, available in online article). In the weighted multiple logistic regressions, consistently no difference in the odds of having either MPR $\geq 80 \%$ or $\mathrm{aMPR} \geq 80 \%$ was found by index insulin type. In the analyses stratified by previous insulin experience, mostly similar results for the primary independent variable were produced by all models except that among insulin-experienced patients, the odds 


\section{TABLE 2}

\begin{tabular}{l|rr|rr|rr|r}
\hline Adherence & \multicolumn{2}{|c|}{$\begin{array}{c}\text { Intermediate- } \\
\text { Acting (NPH) } \\
(\mathbf{n = 1 8 7 )}\end{array}$} & \multicolumn{2}{|c|}{$\begin{array}{c}\text { First-Generation } \\
\text { Long-Acting } \\
(\mathbf{n}=\mathbf{4 , 5 2 2})\end{array}$} & $\begin{array}{c}\text { Second-Generation } \\
\text { Long-Acting } \\
(\mathbf{n}=\mathbf{3 2 5})\end{array}$ & P Value $^{\mathrm{a}}$ \\
\hline MPR, mean (SD) & 0.55 & $(0.27)^{\mathrm{b}}$ & 0.59 & $(0.27)^{\mathrm{c}}$ & 0.68 & $(0.25)^{\mathrm{d}}$ & $<0.0001$ \\
\hline aMPR, mean (SD) & 0.73 & $(0.28)^{\mathrm{b}}$ & 0.78 & $(0.26)^{\mathrm{c}}$ & 0.83 & $(0.23)^{\mathrm{d}}$ & 0.0001 \\
\hline MPR $\geq 0.8, \mathrm{n}(\%)$ & 40 & $(21.39)^{\mathrm{b}}$ & 1,249 & $(27.62)^{\mathrm{b}}$ & 129 & $(39.69)^{\mathrm{c}}$ & $<0.0001$ \\
\hline aMPR $\geq 0.8, \mathrm{n}(\%)$ & 92 & $(49.20)^{\mathrm{b}}$ & 2,711 & $(59.95)^{\mathrm{c}}$ & 218 & $(67.08)^{\mathrm{d}}$ & 0.0004 \\
\hline
\end{tabular}

aP values were based on analysis of variance for continuous variables and on chi-square tests for categorical variables.

$b, c, d$ Means (or proportions) with the same letter were not significantly different (Duncan's multiple range test or chi-square test with type 1 error corrected using Bonferroni).

eTruncated at 1.

$a M P R=$ adjusted medication possession ratio; $M P R=$ medication possession ratio; $N P H=$ neutral protamine Hagedorn; SD = standard deviation.

of having $\mathrm{MPR} \geq 80 \%$ in FGLA insulin users was significantly lower by $33.4 \%$ $(\mathrm{OR}=0.666, \quad 95 \% \quad \mathrm{CI}=0.504-0.880)$ compared with SGLA insulin users.

\section{Discussion}

This study assessed basal insulin adherence over the year following the index prescription among Texas Medicaid enrollees with T2D. Among the included patients, the vast majority were on FGLA insulin (89.8\%) and were naive to any basal insulin (74.9\%). Consistent with previous studies reporting a mean MPR range between 0.44 and 0.69 among basal insulin-naive patients, $15,18,19,21,22,26,27$ this study found suboptimal adherence (0.55-0.68) for all 3 types of basal insulin users when using MPR. It was expected and also consistent with previous research that when using aMPR, patients in each group appeared to be more adherent, compared with when MPR was used. ${ }^{28}$ Among basal insulin-naive patients initiated on NPH or FGLA insulin, previously reported aMPRs ranged from 0.38 to $0.73,,^{16,18-21,23,29}$ whereas for patients with baseline basal insulin experience, aMPR ranged from 0.61 to $0.86 .^{17,20,22}$ The aMPR values $(0.73-0.83)$ in this study, which included basal insulin naive and experienced patients and excluded patients with the poorest adherence (by requiring $\geq 2$ prescriptions of index insulin), fall within the previously reported range.

Very limited evidence is available specifically on adherence to SGLA insulin. One study, Zhou et al. (2018), used PDC to evaluate the effect of an educational patient program on adherence among T2D patients on glargine U300. ${ }^{30}$ Zhou et al. reported that $68.0 \%$ of the program participants $(\mathrm{N}=544)$ and $61.4 \%$ of the nonparticipants $(\mathrm{N}=544$; propensity score matched to participants) were adherent ( $\mathrm{PDC} \geq 80 \%$ ). The adherence rates were higher than that observed among SGLA insulin users in the current study (39.7\% had $\mathrm{MPR} \geq 80 \%)$. Of note, compared with patients in the present study, patients in the Zhou et al. study were mostly covered by commercial insurance, slightly older, and had observably more OHAs (about 2 more OHAs) at baseline.

Even less empirical evidence exists on SGLA insulin adherence compared with other basal insulins, although studies have shown improved regimen convenience and/or patient preference with SGLA insulin. ${ }^{31-33}$ This is further supported by 2 recent reports that suggest better persistence with SGLA insulin versus FGLA insulin over 6 months. ${ }^{34,35}$ Additional support is provided by the current study. In the bivariate comparisons using MPR, SGLA insulin users showed better adherence compared with NPH and FGLA insulin users. Higher adherence to SGLA insulin was maintained when using aMPR, although adherence differences diminished after applying the adjustment factors, since SGLA insulin users, on average, were assigned smaller adjustment factors (Supplementary Table 3, available in online article).

In the main analyses that adjusted for covariates, however, no significant association was found between the odds of adherence and basal insulin type, using either MPR or aMPR. The better odds of having MPR $\geq 80 \%$ in FGLA versus SGLA, observed in the sensitivity analyses, among experienced insulin users and the lack of such effect among insulin-naive users warrant further investigation because it may provide additional justification for prior authorizations for SGLA insulins. ${ }^{36}$ The group sizes in the current stratified analyses were small.

While MPR and aMPR provide consistent results in the main analyses, careful consideration should be given to the limitations of both measures before interpreting the results. A discussion of the methodological considerations was included hereafter, although no formal comparison between MPR and aMPR was performed. One assumption made by traditional MPR is that the recorded days supply accurately represents the "true" length of time supplied, which may not hold in insulin adherence measurement. Inaccuracies may stem from multiple sources such as patients' need for dose adjustments that are not well reflected in prescribers' 


\section{TABLE 3 Logistic Regression Analyses Comparing the Odds of Adherence Among Basal Insulins $(\mathrm{N}=5,034)$}

\begin{tabular}{|c|c|c|c|c|c|c|c|c|}
\hline \multirow[b]{2}{*}{ Variables } & \multicolumn{4}{|c|}{$a M P R \geq 0.8$} & \multicolumn{4}{|c|}{$M P R \geq 0.8$} \\
\hline & OR & \multicolumn{2}{|c|}{$95 \% \mathrm{Cl}$} & $P$ Value & OR & \multicolumn{2}{|c|}{$95 \% \mathrm{Cl}$} & $P$ Value \\
\hline \multicolumn{9}{|l|}{ Insulin type ${ }^{a}$} \\
\hline $\mathrm{NPH}$ & 0.924 & 0.622 & 1.372 & 0.6955 & 0.720 & 0.463 & 1.119 & 0.1441 \\
\hline First-generation long-acting & 1.158 & 0.893 & 1.501 & 0.2692 & 0.815 & 0.633 & 1.049 & 0.1127 \\
\hline
\end{tabular}

Age, years ${ }^{a}$

\begin{tabular}{|c|c|c|c|c|c|c|c|c|}
\hline $18-39$ & 0.753 & 0.612 & 0.925 & 0.0069 & 0.651 & 0.512 & 0.828 & 0.0005 \\
\hline $40-49$ & 1.102 & 0.913 & 1.329 & 0.3119 & 0.806 & 0.660 & 0.985 & 0.0348 \\
\hline $50-59$ & 0.975 & 0.831 & 1.145 & 0.7599 & 0.909 & 0.769 & 1.075 & 0.2648 \\
\hline Female $^{\mathrm{a}}$ & 0.936 & 0.826 & 1.060 & 0.2982 & 0.866 & 0.756 & 0.991 & 0.0368 \\
\hline Pre-index use of basal insulin ${ }^{a}$ & 1.982 & 1.708 & 2.300 & $<0.0001$ & 1.541 & 1.330 & 1.785 & $<0.0001$ \\
\hline Pre-index OHA adherence & 1.013 & 1.010 & 1.015 & $<0.0001$ & 1.015 & 1.012 & 1.017 & $<0.0001$ \\
\hline Pre-index use of comorbid medications ${ }^{a}$ & 1.054 & 0.934 & 1.190 & 0.3934 & 0.934 & 0.819 & 1.066 & 0.3130 \\
\hline Baseline total number of medications & 1.057 & 1.044 & 1.071 & $<0.0001$ & 1.066 & 1.052 & 1.079 & $<0.0001$ \\
\hline Post-index number of OHAs & 0.911 & 0.856 & 0.970 & 0.0036 & 0.915 & 0.854 & 0.979 & 0.0105 \\
\hline Post-index use of bolus insulina & 1.179 & 1.044 & 1.332 & 0.0081 & 1.249 & 1.093 & 1.427 & 0.0011 \\
\hline Post-index use of GLP-1 RA ${ }^{a}$ & 1.347 & 1.110 & 1.634 & 0.0025 & 1.244 & 1.025 & 1.510 & 0.0271 \\
\hline
\end{tabular}

Note: Model fit statistics for outcome of $a M P R \geq 0.8$ : Likelihood ratio $=420.5070, d f=13 ; P<0.0001 ;$ for outcome of $M P R \geq 0.8$ : Likelihood ratio =398.5705, df =13; $P<0.0001$.

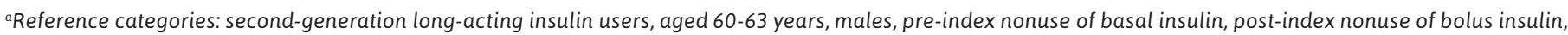
post-index nonuse of GLP-1 RA, and pre-index nonuse of medications for comorbid hypertension and dyslipidemia.

aMPR = adjusted medication possession ratio; $C l=$ confidence interval; $G L P-1$ RA=glucagon-like peptide-1 receptor agonist; $M P R=$ medication possession ratio; $\mathrm{NPH}=$ neutral protamine Hagedorn; $\mathrm{OHA}=$ oral hypoglycemic agent; $\mathrm{OR}=$ odds ratio.

directions and pharmacists' variation in calculating days supply (e.g., whether to round up or round down days supply and whether the minimum dispensing unit is a whole box or an individual pen). Although, for insulin products of different package sizes, it would be expected that the mean number of days supplied would vary because of the different mean quantities dispensed. One previous study showed that the mean recorded days supply was almost invariably close to 30 days (Supplementary Table 3, available in online article). ${ }^{15}$ Therefore, traditional MPR may underestimate adherence to insulin products of larger package sizes. ${ }^{28}$

"True" days supply should be determined by the total quantity dispensed and a patient's daily required dosage. As mentioned in the Methods section, the aMPR method may mitigate the effect of inaccurately recorded days supply at the group level. Unlike previous studies reporting longer refill intervals for products of larger package sizes, ${ }_{15,16}$ products of larger package sizes had either longer or shorter average refill intervals in this study (Supplementary Table 3 , available in online article). This may be because previous studies compared therapeutically similar products (e.g., glargine U100 vs. detemir), whereas products included in this study were therapeutically different, and average patients' insulin needs may vary across groups. For example, in this study, despite its relatively larger package size, glargine U300 (1,350 units per package) had shorter between-refill intervals compared with any insulin product delivered via vials (1,000 units per package) possibly because patients initiated on concentrated insulin had greater average insulin needs. Although aMPR has advantages, one disadvantage is that the mean or median time between refills can be affected by other factors, such as patients' insulin use behaviors.

Unfortunately, claims data cannot be used to distinguish whether the interval variation is because of varying insulin needs or patient behaviors. In the example of glargine U300 users, in addition to greater average insulin needs, the shorter average between-refill intervals can also be possibly explained by relatively more prescriptions refilled on time, compared with users of other basal insulins. In 
fact, given that suboptimal insulin adherence is common among patients with diabetes ${ }^{37}$ the average time between refills may overestimate the actual number of days supplied. Thus, using aMPR may lead to overall overestimation of actual adherence regardless of insulin type, which was also suggested by 1 previous study. ${ }^{28}$

Further, using aMPR is also likely to bias adherence comparisons when patients' insulin-consuming behaviors differ by group, since patient groups frequently delaying refills are "compensated" for delaying with larger adjustment factors. In this study, SGLA insulin users were likely to have had different insulin needs and different insulin use behaviors, compared with other basal insulin users.

It should also be noted that, since unique adjustment factors are calculated for each group using the same insulin product, aMPR makes adjustments only at the group level; no individual-level adjustment is made within a patient group on the same insulin. However, at the individual level, insulin supply and needs vary, and this variation is not captured by MPR or aMPR. Regarding insulin supply, even within a patient group on the same insulin, actual dispensing patterns may vary and lead to varying accuracy of recorded days supply. For example, allowing an individual pen to be dispensed, rather than a whole package, as the minimal dispensing unit may enable higher accuracy of recorded days supply. ${ }^{38}$ Regarding insulin needs, 1 patient may have greater up-titration needs, which is not accounted for at the time of dispensing. Thus, an overestimation of days supply may occur due to the fewer "true" days supplied. For example, a patient may use all of the insulin supplied in 20 days rather than the 30 days that was documented during the time of dispensing. Failure of MPR and aMPR to capture varying insulin supply and needs at the individual level means that, when using either measure, associations between adherence and factors examined may be confounded by insulin supply and need factors. For the aMPR method, a possibility to mitigate such confounding is to calculate adjustment factors within homogeneous subgroups consisting of individuals with similar insulin supply and needs. Yet, it is practically challenging to identify such subgroups, and it may not always be possible to obtain large enough subgroups.

In the absence of other adherence measures, MPR and aMPR should be used while considering the previously mentioned limitations. Following are some recommendations to consider when using MPR and/or aMPR to measure insulin adherence:

1. When choosing between MPR and aMPR, aMPR may be better suited for insulin adherence measurement, since it takes into account the inaccuracies of recorded days supply, although one should be aware that the adjustment factor in aMPR can be affected by patients' actual adherence, and the absolute values of aMPR may generally overestimate adherence.

2. If aMPR is used to compare adherence, it is beneficial to have patient groups with similar insulin-consuming patterns to prevent biased adjustments to different groups. Previous studies have employed techniques such as propensity score matching to obtain patient groups of similar baseline characteristics and potentially similar baseline medication-taking behaviors..$^{15,17-23}$ However, patient counterparts using different insulin products may not always be available for matching. In Texas Medicaid, while all NPH and FGLA insulin products (except Basaglar [a glargine U100 product]) studied were classified as preferred agents, SGLA insulin products (glargine U300 and degludec) were nonpreferred and required prior authorization. ${ }^{36}$ In addition, both SGLA insulin products included were concentrated forms and more likely to be initiated among patients requiring large doses. ${ }^{6}$ Such a factual patient selection due to access constraints and different indications may lead to fundamental patient differences in SGLA insulin users compared with other basal insulin users. Considering the risk of biased adjustments made by aMPR, it is advisable to report both MPR and aMPR results.

3. When using either MPR or aMPR, caution needs to be applied when examining factors (other than insulin type) associated with adherence, since there is a risk of confounding by individual insulin needs and pharmacy dispensing patterns.

Bearing in mind the limitations of MPR and aMPR, this study did not find significantly better adherence favoring SGLA insulin compared with other basal insulins among Texas Medicaid patients with T2D, controlling for covariates. Thus, regarding basal insulin selection, clinicians caring for Texas Medicaid patients may focus on other more established clinical benefits (e.g., reduced risk of hypoglycemia) associated with SGLA insulin instead of the previously speculated adherence benefits. ${ }^{6,39}$

\section{LIMITATIONS}

This study has several limitations. First, as an observational study, selection bias may be present, as suggested by the baseline characteristics. Sensitivity analyses using PSW in addition to covariate adjustment were performed to mitigate the bias, and our main findings were robust. However, since most NPH insulin users had vial delivery, and all SGLA insulin users had pen delivery, this factor was not likely to achieve balance or to be controlled for in the regressions. Previous studies generally suggested better adherence 
favoring pens, ${ }^{26,40}$ although 1 Medicaid study found similar or better adherence with vials. ${ }^{41}$

Second, the logistic regression analyses performed in this study were underpowered, mainly because of the imbalanced group sizes across the 3 types of basal insulins. It is likely that association between insulin type and adherence existed but went undetected as a result of an increased risk of type 2 error.

Third, while capping MPR at 1 and using 0.8 as a threshold is conventional for MPR, no validated threshold exists for aMPR. Capping aMPR at 1 and using 0.8 as a cutoff were mostly arbitrary and may have further reduced power.

Finally, the study results may not be generalizable beyond Texas Medicaid adult enrollees. The patients included in this study were mainly patients with disability and/or of a low socioeconomic status, and there was no copay for prescriptions.

\section{Conclusions}

No association was found between basal insulin adherence and insulin type after controlling for covariates in this study. This suggests that SGLA insulin possibly did not provide additional adherence benefits to Texas Medicaid basal insulin users, although this finding needs further confirmation because of the study's limitations. Caution needs to be shown when using MPR or aMPR for insulin adherence measurement, since limitations exist with both methods. Formal validation of MPR and aMPR in measuring insulin adherence is needed.

\section{DISCLOSURES}

No specific funding was received for this manuscript. The authors report no potential conflicts of interest.

Part of the data from this study was presented as posters at the American Pharmacists Association 2020 Annual Meeting \& Exposition,
March 20-23, 2020, in National Harbor, $\mathrm{MD}$, and at the International Society for Pharmacoeconomics and Outcomes Research 2020 Conference, May 16-20, 2020, in Orlando, FL.

\section{ACKNOWLEDGMENTS}

The authors thank Texas Medicaid for the use of its data to conduct this study.

\section{REFERENCES}

1. Menke A, Casagrande S, Geiss L, Cowie CC. Prevalence of and trends in diabetes among adults in the United States, 19882012. JAMA. 2015;314(10):1021-29.

2. Geiss LS, Kirtland K, Lin J, et al. Changes in diagnosed diabetes, obesity, and physical inactivity prevalence in U.S. counties, 2004-2012. PLoS One. 2017;12(3):e0173428.

3. Geiss LS, Wang J, Cheng YJ, et al. Prevalence and incidence trends for diagnosed diabetes among adults aged 20 to 79 years, United States, 1980-2012. JAMA. 2014;312(12):1218-26.

4. Centers for Disease Control and Prevention. National diabetes statistics report 2020. Accessed September 22, 2020. https://www.cdc.gov/diabetes/ pdfs/data/statistics/national-diabetesstatistics-report.pdf

5. American Diabetes Association.

Economic costs of diabetes in the U.S. in 2017. Diabetes Care. 2018;41(5):917-28.

6. American Diabetes Association. 9. Pharmacologic approaches to glycemic treatment: standards of medical care in diabetes-2020. Diabetes Care. 2020;43(Suppl 1):S98-S110.

7. Selvin E, Parrinello CM, Daya N, Bergenstal RM. Trends in insulin use and diabetes control in the U.S.: 1988-1994 and 1999-2012. Diabetes Care. 2016;39(3):e33-e35.

8. Garber AJ, Handelsman Y, Grunberger G, et al. Consensus statement by the American Association of Clinical Endocrinologists and American College of Endocrinology on the comprehensive type 2 diabetes management algorithm2020 executive summary. Endocr Pract. 2020;26(1):107-39.
9. Pettus J, Santos Cavaiola T, Tamborlane WV, Edelman S. The past, present, and future of basal insulins. Diabetes Metab Res Rev. 2016;32(6):478-96.

10. Cahn A, Miccoli R, Dardano A, Del Prato S. New forms of insulin and insulin therapies for the treatment of type 2 diabetes. Lancet Diabetes Endocrinol. 2015;3(8):638-52.

11. Doggrell SA, Chan V. Adherence to insulin treatment in diabetes: can it be improved? J Diabetes. 2015;7(3):315-21.

12. Cramer JA. A systematic review of adherence with medications for diabetes. Diabetes Care. 2004;27(5):1218-24.

13. Peyrot M, Barnett AH, Meneghini LF, Schumm-Draeger PM. Insulin adherence behaviours and barriers in the multinational Global Attitudes of Patients and Physicians in Insulin Therapy study. Diabet Med. 2012;29(5):682-89.

14. Stolpe S, Kroes MA, Webb N, Wisniewski T. A systematic review of insulin adherence measures in patients with diabetes. J Manag Care Spec Pharm. 2016;22(11):1224-46. doi: 10.18553/ jmcp.2016.22.11.1224

15. Baser O, Bouchard J, DeLuzio T, Henk H, Aagren M. Assessment of adherence and healthcare costs of insulin device (FlexPen $囚$ ) versus conventional vial/ syringe. Adv Ther. 2010;27(2):94-104.

16. Buysman E, Conner C, Aagren M, Bouchard J, Liu F. Adherence and persistence to a regimen of basal insulin in a pre-filled pen compared to vial/syringe in insulin-naïve patients with type 2 diabetes. Curr Med Res Opin. 2011;27(9):1709-17.

17. Xie L, Zhou S, Wei W, Gill J, Pan C, Baser O. Does pen help? A real-world outcomes study of switching from vial to disposable pen among insulin glargine-treated patients with type 2 diabetes mellitus. Diabetes Technol Ther. 2013;15(3):230-36.

18. Grabner M, Chu J, Raparla S, Quimbo R, Zhou S, Conoshenti J. Clinical and economic outcomes among patients with diabetes mellitus initiating insulin glargine pen versus vial. Postgrad Med. 2013;125(3):204-13. 
19. Xie L, Wei W, Pan C, Du J, Baser O. A real-world study of patients with type 2 diabetes initiating basal insulins via disposable pens. Adv Ther. 2011;28(11):1000-11.

20. Miao R, Wei W, Lin J, Xie L, Baser O. Does device make any difference? A real-world retrospective study of insulin treatment among elderly patients with type 2 diabetes. J Diabetes Sci Technol. 2014;8(1):150-58.

21. Wang L, Wei W, Miao R, Xie L, Baser O. Real-world outcomes of U.S. employees with type 2 diabetes mellitus treated with insulin glargine or neutral protamine Hagedorn insulin: a comparative retrospective database study. BMJ Open. 2013;3(4):1-9.

22. Wei W, Zhou S, Miao R, et al. Much ado about nothing? A real-world study of patients with type 2 diabetes switching basal insulin analogs. Adv Ther. 2014;31(5):539-60.

23. Baser O, Tangirala K, Wei W, Xie L. Real-world outcomes of initiating insulin glargine-based treatment versus premixed analog insulins among U.S. patients with type 2 diabetes failing oral antidiabetic drugs. Clin Outcomes Res. 2013;5(1):497-505.

24. Happe LE, Brown JD, Gatwood J, Schneeweiss S, Wang S. Application of a graphical depiction of longitudinal study designs to managed care pharmacy research. J Manag Care Spec Pharm. 2020;26(3):268-74. doi: 10.18553/ jmcp.2020.26.3.268

25. Karve S, Cleves MA, Helm M, Hudson TJ, West DS, Martin BC. An empirical basis for standardizing adherence measures derived from administrative claims data among diabetic patients. Med Care. 2008;46(11):1125-33.

26. Asche CV, Shane-Mcwhorter L, Raparla S. Health economics and compliance of vials/syringes versus pen devices: a review of the evidence. Diabetes Technol Ther. 2010;12(Suppl 1):S101-S108.
27. Fabunmi R, Nielsen LL, Quimbo R, et al. Patient characteristics, drug adherence patterns, and hypoglycemia costs for patients with type 2 diabetes mellitus newly initiated on exenatide or insulin glargine. Curr Med Res Opin. 2009;25(3):777-86.

28. Stephenson JJ, Bae JP, Raval AD, Kern DM. Bridging the gap between selfreported and claims-derived adherence measures for basal insulin among patients with type 2 diabetes mellitus. Adv Ther. 2019;36(1):118-30.

29. Chandran A, Bonafede MK, Nigam S, Saltiel-Berzin R, Hirsch LJ, Lahue BJ. Adherence to insulin pen therapy is associated with reduction in healthcare costs among patients with type 2 diabetes mellitus. Am Health Drug Benefits. 2015;8(3):148-58.

30. Zhou FL, Yeaw J, Karkare SU, DeKoven M, Berhanu P, Reid T. Impact of a structured patient support program on adherence and persistence in basal insulin therapy for type 2 diabetes. BMJ Open Diabetes Res Care. 2018;6(1):e000593.

31. Warren ML, Brod M, Håkan-Bloch J, Sparre T, Chaykin LB. Patient-reported outcomes from a randomized, crossover trial comparing a pen injector with insulin degludec versus a pen injector with insulin glargine U100 in patients with type 2 diabetes. Curr Med Res Opin. 2019;35(9):1623-29.

32. Warren ML, Chaykin LB, Jabbour S, et al. Insulin degludec 200 Units $/ \mathrm{mL}$ is associated with lower injection frequency and improved patient-reported outcomes compared with insulin glargine 100 Units/ $\mathrm{mL}$ in patients with type 2 diabetes requiring high-dose insulin. Clin Diabetes. 2017;35(2):90-95.

33. Wieringa TH, de Wit M, Twisk JW, Snoek FJ. Improved diabetes medication convenience and satisfaction in persons with type 2 diabetes after switching to insulin glargine $300 \mathrm{U} / \mathrm{mL}$ : results of the observational OPTIN-D study. BMJ Open Diabetes Res Care. 2018;6(1):e000548.
34. IQVIA, Forte Information Resources. LVBCH type 2 diabetes report. 7th ed. Sanofi Managed Care Digest Series. 2019. Accessed September 22, 2020. http:// www.lvbch.com/upload/file/LVBCH\%20 2019\%20Diabetes\%20Report.pdf

35 IQVIA, Forte Information Resources. LVBCH type 2 diabetes report. 6th ed. Sanofi Managed Care Digest Series. 2018. Accessed September 22, 2020. http:// www.lvbch.com/upload/file/LVBCH\%20 2018\%20Diabetes\%20Report_FINAL.pdf

36. Texas Health and Human Services. Medicaid pharmacy prior authorization \& preferred drug list. Accessed September 22, 2020. https://www.txvendordrug. com/sites/txvendordrug/files/docs/ formulary/2020-0130-preferred-druglist.pdf

37. Peyrot M, Rubin RR, Kruger DF, Travis LB. Correlates of insulin injection omission. Diabetes Care. 2010;33(2):240-45.

38. U.S. ex rel Rahimi v Walgreens Boots Alliance Inc. U.S. District Court, Southern District of New York. No. 15-05686. 15 Civ. 5686 (PAC). Accessed September 22, 2020. https://www.justice.gov/usao-sdny/ press-release/file/1124501/download

39. Zhou FL, Ye F, Berhanu P, et al. Realworld evidence concerning clinical and economic outcomes of switching to insulin glargine 300 units/mL vs other basal insulins in patients with type 2 diabetes using basal insulin. Diabetes Obes Metab. 2018;20(5):1293-97.

40. Guerci B, Chanan N, Kaur S, JassoMosqueda JG, Lew E. Lack of treatment persistence and treatment nonadherence as barriers to glycaemic control in patients with type 2 diabetes. Diabetes Ther. 2019;10(2):437-49.

41. Pawaskar MD, Camacho FT, Anderson RT, Cobden D, Joshi AV, Balkrishnan R. Health care costs and medication adherence associated with initiation of insulin pen therapy in Medicaid-enrolled patients with type 2 diabetes: a retrospective database analysis. Clin Ther. 2007;29 Spec No:1294-305. 